\title{
Prevalence of SARS-CoV-2 in Conjunctival Swab Samples Among Patients Presenting with Conjunctivitis During the COVID-19 Pandemic
}

\author{
Sezen Karakus $\mathbb{D}^{\prime}$ \\ James Foster' \\ Xi Dai $\mathbb{D D}^{1,2}$ \\ Anthony Gonzales' \\ Xi Zhu ${ }^{1,3}$ \\ Charles Eberhart' \\ William Hsu ${ }^{1,4}$
}

'Wilmer Eye Institute, Johns Hopkins University School of Medicine, Baltimore, MD, USA; ${ }^{2}$ Johns Hopkins University School of Medicine, Baltimore, MD, USA; ${ }^{3}$ Department of Biology, Public Health Studies, Krieger School of Arts and Sciences, Johns Hopkins University, Baltimore, MD, USA; ${ }^{4}$ Department of Molecular and Cell Biology, Krieger School of Arts and Sciences, Johns Hopkins University, Baltimore, MD, USA
Correspondence: Sezen Karakus Wilmer Eye Institute, Johns Hopkins University School of Medicine, 600

N. Wolfe Street, Maumenee 74I,

Baltimore, MD, 21287 , USA

Tel +l 4109550128

Fax +1 4106141670

Email skarakus@jhmi.edu
Purpose: To investigate the prevalence of SARS-CoV-2-associated conjunctivitis among patients presenting with conjunctivitis to ophthalmology clinics in the absence of respiratory symptoms suggesting COVID-19 during the first year of the pandemic.

Patients and Methods: This prospective, observational, cross-sectional study enrolled patients aged 18 years and older who presented with acute conjunctivitis between May 2020 and May 2021. After reviewing demographics, ocular and systemic symptoms, a slit lamp examination was performed. Five samples were collected (conjunctival swab from each eye, nasal swab from each nostril, and a nasopharyngeal swab) in separate tubes for a reverse transcription-polymerase chain reaction test for SARS-CoV-2. Patients were called at 1 and 2 weeks to follow up on their ocular and systemic symptoms and collect information about conjunctival adenovirus test results and COVID-19 test results if performed outside of the study. Results: A total of 36 patients were enrolled. The most common ocular symptom was redness (35/36, 97\%), and 14 patients (39\%) had symptoms in both eyes at the time of the presentation. SARS-CoV-2 RNA was not detected in any samples collected during the study visit $(95 \%$ confidence interval $[\mathrm{CI}]$ from 0 to 0.08$)$. None of the study participants were diagnosed with COVID-19 following conjunctivitis during the 2-week follow-up period. We found that 25 patients had a conjunctival adenovirus test done on the day of their study visit, 9 of which reported positive results.

Conclusion: Our data suggest that it may not be valuable to obtain routine testing of SARSCoV-2 in patients presenting with conjunctivitis in the absence of COVID-19.

Keywords: adenovirus, conjunctivitis, coronavirus, COVID-19, SARS-CoV-2

\section{Introduction}

Severe acute respiratory syndrome coronavirus 2 (SARS-CoV-2) is a highly contagious RNA virus causing a respiratory infectious disease called Coronavirus Disease 2019 (COVID-19). COVID-19 is mainly transmitted from person to person via respiratory droplets and typically presents with fever, cough, and shortness of breath. Conjunctivitis has been reported as a rare finding of COVID-19, reported between 0.8 and $32 \%$; however, a meta-analysis concluded that the overall prevalence of conjunctivitis in patients with confirmed COVID-19 is about $1.1 \% .^{1-4}$ The possibility of direct conjunctival inoculation has been speculated as a source of infection since there have been multiple confirmed cases of COVID-19 presenting with conjunctivitis, and the presence of viral transcripts in ocular secretions of patients with conjunctivitis. $^{5-9}$ 
Previous studies investigating the prevalence of SARSCoV-2 in ocular specimens included patients with confirmed or suspected cases of COVID-19. ${ }^{5-9}$ A few case reports showed the presence of SARS-CoV-2 RNA in otherwise healthy patients presenting with follicular conjunctivitis, suggesting that conjunctivitis could be the only presenting sign of underlying infection. ${ }^{10,11}$ However, it is unclear how prevalent SARS-CoV-2 associated conjunctivitis is among patients presenting only with conjunctivitis to the ophthalmology clinics without having any respiratory symptoms and whether extra cautions are necessary for this patient group in ophthalmology practices during the pandemic. Compounding the emerging reports of the presence of viral transcripts at the ocular surface, the initiation of the pandemic response (stay-at-home orders, lockdowns) came a perceived increase in emergency room visits for conjunctivitis within our clinics. While not quantified here, this observation provided the impetus for subsequent investigations. Therefore, we aimed to determine the prevalence of SARS-CoV-2 associated conjunctivitis among patients presenting to the ophthalmology clinics with suspected viral conjunctivitis. We also aimed to investigate whether conjunctivitis could be an early sign of COVID-19.

\section{Patients and Methods}

\section{Study Design and Participants}

This prospective cross-sectional observational study was approved by the Johns Hopkins University Institutional Review Board and adhered to the tenets of the Declaration of Helsinki. Patients aged 18 years or older presenting with acute conjunctivitis to the Wilmer Eye Institute, Johns Hopkins University, Baltimore, Maryland, were recruited between May 2020 and May 2021. During the study period, all patients were being screened for COVID-19 before being accepted to the outpatient ophthalmology clinics. Patients with respiratory symptoms suggesting COVID-19 were being admitted to the emergency department and assessed after a formal COVID-19 test. Therefore, they were not included in this study. In addition, patients who presented with red-eye symptoms due to other ocular pathologies such as glaucoma, known chemical or environmental exposure, foreign body, trauma, intraocular inflammation, or infection were excluded.

\section{Study Procedures}

After obtaining informed consent, adhering to the tenets of the declaration of Helsinki, participants were administered a standardized questionnaire reviewing their demographics, ocular and systemic health, and symptoms at baseline. Slit-lamp examination and external photography of eyes were performed to characterize and document pathological changes. Five swabs were taken: a conjunctival swab from each eye, a nasal swab from each nostril, and a nasopharyngeal swab. A separate sterile polyester swab with a plastic shaft was used for each swab and immediately placed into separate sterile tubes containing an RNA stabilization solution (DNA/RNA Shield ${ }^{\mathrm{TM}}$, Zymo Research Corporation, Irvine, CA). The conjunctival swab was taken by putting a swab in the lower conjunctival fornix without using topical anesthetic drops. For the nasal swab, a swab was inserted about one inch into the nostril until resistance was met at the turbinates. For the nasopharyngeal swab, a swab was inserted through one of the nostrils parallel to the palate until it contacted the nasopharynx. The swabs were left in place for several seconds to absorb secretions and slowly removed while rotating them. Gloves were changed after collecting each sample to avoid cross-contamination.

All patients were contacted via phone twice, at seven and fourteen days after the initial visit, to review their ocular and systemic symptoms and laboratory test results such as conjunctival adenovirus test or COVID-19 test if it was done outside of the study.

\section{Specimen Testing and Laboratory Methods}

Specimens collected via conjunctival, nasal, and nasopharyngeal swabs were delivered to the extraction site submerged in DNA/RNA Shield (Zymo Research Corporation, Irvine, CA) to protect the quality of viral RNA while inactivating the virus. Samples were then stored at $-20^{\circ} \mathrm{C}$ until required. Polymerase Chain Reaction (PCR) assays were utilized to test the specimens as it was a widely accepted and effective technique for this purpose. ${ }^{12}$ Viral RNA was extracted using the Quick-RNA Viral Kit (Zymo Research Corporation, Irvine, CA) according to the manufacturer's instructions. Complementary DNA (cDNA) synthesis and reverse transcription (RT)-PCR were carried out using the 1-Step Quantitative Reverse Transcription PCR (BioRad Laboratories, Inc., Hercules, CA) according to the manufacturer's instructions on the Applied Biosystems QuantStudio 3 Real-Time PCR System (Thermo Fisher Scientific Inc., Waltham, MA). Extraction and quantification were assayed using the United States Centers for Disease Control and Prevention (CDC) recommended 
PCR probe set for 2019-nCoV_N1, N2, and Human RNase P. Extraction efficiency and copy number were assayed against a randomly chosen duplicate sample which had been spiked with $1 \mu \mathrm{L}$ of heat-inactivated viral particles (BEI resources, NR-52286). Quantitative synthetic RNA (BEI resources, NR-52350) was used to derive the sensitivity for assaying viral load; samples with a cT $>32 \quad(<1$ viral genome in $400 \mu \mathrm{L}$ sample $)$ were deemed negative. Human corneal epithelial RNA extracted before 2019 was used as a negative, noninfected control.

\section{Statistical Analysis}

Demographic and baseline characteristics were summarized using descriptive analysis. Continuous variables were reported as mean and standard deviation, and categorical/binary variables were reported as numbers and percentages. The primary outcome variable was the proportion of conjunctival samples that tested positive for SARS-CoV-2. Secondary outcome variables included proportions or nasal and nasopharyngeal tested positive for SARS-CoV-2 and rate of development COVID-19 in the study population. The Wilson score method was used to calculate the $95 \%$ confidence interval (CI) for proportions. STATA 16 was used for analysis (StataCorp LLC, College Station, TX, USA).

\section{Results}

A total of 36 patients with conjunctivitis were enrolled in the study. Table 1 displays the patient demographic and clinical characteristics. The most common ocular symptom was redness $(35 / 36,97 \%)$, and 14 patients $(39 \%)$ had symptoms in both eyes at the time of the presentation. Seventeen patients $(17 / 36,47 \%)$ reported seasonal allergies. Ten patients $(10 / 36,28 \%)$ reported being given a topical antibiotic or a combination of antibiotic and steroid eye drops prescribed by the referring physician. Three patients $(3 / 36,8 \%)$ reported using anti-allergy eye drops. None of these patients reported using these eye drops on the day of the sample collection. Conjunctival samples were collected from all 36 patients; however, three patients declined to provide nasal and nasopharyngeal swabs, and 13 agreed to provide nasal samples but declined nasopharyngeal swabs, ie, nasal samples were collected in 33, and nasopharyngeal samples were collected in 23 patients. SARS-CoV-2 RNA was not detected in any of the samples collected during the study visit. The $95 \%$ confidence interval for the zero-occurrence rate of
Table I Demographics and Clinical Characteristics of Patients Presented with Acute Conjunctivitis to Ophthalmology Clinics Between May 2020 and May 202I

\begin{tabular}{|c|c|}
\hline Variables & Patients $(n=36)$ \\
\hline \multicolumn{2}{|l|}{ Age (years) } \\
\hline Mean (SD) & $42.6(17.0)$ \\
\hline Range & $18-85$ \\
\hline Sex (male), n (\%) & $18(50)$ \\
\hline \multicolumn{2}{|l|}{ Ocular Symptoms, n (\%) } \\
\hline Redness & $35(97)$ \\
\hline Swelling & $29(8 \mathrm{I})$ \\
\hline Tearing & $28(78)$ \\
\hline Irritation & $28(78)$ \\
\hline Crusting & $23(64)$ \\
\hline Itching & $21(58)$ \\
\hline Bilateral Symptoms, n (\%) & $14(39)$ \\
\hline \multicolumn{2}{|l|}{ Slit Lamp Findings, n (\%) } \\
\hline Injection & 34 (94) \\
\hline Follicles & $32(89)$ \\
\hline Eyelid edema & $14(39)$ \\
\hline Punctate epithelial erosions & $14(39)$ \\
\hline Chemosis & $10(28)$ \\
\hline Tearing & 7 (19) \\
\hline Subconjunctival hemorrhage & $2(6)$ \\
\hline \multicolumn{2}{|l|}{ SARS-CoV-2 RNA PCR, n (\%) } \\
\hline Conjunctiva positive & $0 / 36(0)$ \\
\hline Nasal positive & $0 / 33(0)$ \\
\hline Nasopharyngeal positive & $0 / 23(0)$ \\
\hline Adenovirus NAT Test, n (\%) & $25(70)$ \\
\hline Positive & $9 / 25(36)$ \\
\hline
\end{tabular}

Notes: Continuous variables were reported as mean and standard deviation (SD), and categorical/binary variables were reported as numbers and percentages.

Abbreviations: SARS-CoV-2, Severe acute respiratory syndrome coronavirus 2; RNA, ribonucleic acid; PCR, polymerase chain reaction; NAT, nucleic acid test.

viral RNA presence in conjunctival samples was estimated between 0 and 0.08 .

Out of the 36 patients, 25 responded to follow-up calls after the baseline visit. Based on the collected data, the mean (SD) duration of conjunctivitis symptoms was 15.1 (8.9) days. Twenty-five patients had a conjunctival adenovirus test done at the Johns Hopkins Microbiology Laboratory on the same day of the study visit, 9 of which were positive. Fourteen of the remaining 27 patients (16 with negative adenovirus test and 11 without an adenovirus test) reported seasonal allergies (14/27, 52\%), while five of them had bilateral conjunctivitis $(5 / 27$, $18 \%)$. Five patients later developed systemic symptoms that could be associated with COVID-19, including 
headache $(n=2)$, cough $(n=2)$, and muscle ache $(n=1)$. Two out of five symptomatic patients reported being tested for COVID-19, both with negative test results. Two other patients with no systemic symptoms reported having COVID-19 testing for screening purposes with negative test results during the follow-up period. None of the study patients were diagnosed with COVID-19 during the twoweek follow-up period.

\section{Discussion}

This study was conducted to determine whether SARSCoV-2 was a causative agent of conjunctivitis in patients with conjunctivitis presenting to the ophthalmology clinics without having respiratory symptoms indicating COVID19 during the first year of the COVID-19 pandemic. Between May 2020 and May 2021, we enrolled 36 patients with conjunctivitis, and none of the patients tested positive for SARS-CoV-2. To our knowledge, the prevalence of SARS-CoV-2 associated conjunctivitis among conjunctivitis cases presenting primarily to ophthalmology clinics without having respiratory symptoms has not been previously reported. Our data suggested that the frequency of SARS-CoV-2 infection among patients presenting with conjunctivitis symptoms in the absence of COVID-19 is possibly close to zero and unlikely to be more than $8 \%$. Furthermore, none of the study participants in our sample were diagnosed with COVID-19 following conjunctivitis during the 2-week follow-up period.

Conjunctivitis is the most commonly reported ocular manifestation of COVID-19, with a prevalence ranging between $0.8 \%$ and $32 \% .^{1,8,13-15}$ A few case reports indicated that conjunctivitis could be the presenting sign or the only sign of COVID-19; ${ }^{10,11,16}$ therefore, it was recommended that ophthalmologists remain vigilant when a patient presents with conjunctivitis during the pandemic. ${ }^{17}$ However, clinical conjunctivitis may not represent infection of the conjunctiva as it may be an immune response in the setting of systemic disease. In fact, some studies demonstrated that ocular samples from patients with conjunctivitis and ongoing COVID-19 did not always yield positive test results. ${ }^{8}$ Viral RNA presence on the ocular surface was reported as between 0 and $29 \%$ in patients diagnosed with COVID-19 regardless of the presence of conjunctivitis. ${ }^{16,18-20}$ Studies suggest that SARS-CoV-2 viral RNA is more likely to be found in ocular secretions of COVID-19 patients who had conjunctivitis symptoms, although it was also found in patients with no ocular findings in a few cases. ${ }^{21}$ Even though previous reports suggest that ophthalmologists should suspect SARS-CoV-2 infection in any patient presenting with acute conjunctivitis, to our best knowledge, how commonly this occurs in patients presenting with acute conjunctivitis has never been investigated. Within our limited study population, we did not find any patients with conjunctival samples positive for SARS-CoV-2, suggesting that standard red-eye precautions are sufficient in ophthalmology clinics during the pandemic and routine testing for SARS-CoV-2 is not valuable.

Acute conjunctivitis is estimated to affect 20 million people per year in the United States. ${ }^{22}$ Viral conjunctivitis is the most common reason for infectious conjunctivitis, accounting for up to $80 \%$ of acute conjunctivitis cases. ${ }^{23}$ Among viral etiologies, adenovirus is the most common causative agent, reported in $65 \%$ to $90 \%$ of viral conjunctivitis cases. ${ }^{24}$ Other viruses causing acute follicular conjunctivitis include herpes simplex virus (HSV) and varicella zoster virus (VZV), which are not as contagious as adenovirus. Enterovirus 70 and a variant of coxsackievirus A24 were previously responsible for epidemic hemorrhagic conjunctivitis cases. ${ }^{25,26}$ When it comes to coronavirus species other than SARS-CoV-2, human coronavirus NL63, SARS-CoV, and Middle East Respiratory Syndrome (MERS)-related coronavirus, were previously reported to cause conjunctivitis, albeit very uncommonly. ${ }^{27}$ In this study, we aimed to identify SARS$\mathrm{CoV}-2$ associated conjunctivitis cases; therefore, we did not investigate other possible causes of conjunctivitis as part of our study. However, some patients were tested for adenovirus outside of the study $(\mathrm{n}=25)$, and we collected clinical information regarding their test results. Interestingly, only nine patients $(9 / 25,36 \%)$ tested positive for adenovirus. This was below the average reported by literature if we assumed that all cases were due to an infectious etiology. Some of the enrolled patients may have had conjunctivitis due to non-infectious etiologies such as allergic conjunctivitis or dry eye. Allergic conjunctivitis is the most common non-infectious etiology. ${ }^{28}$ In fact, $52 \%$ of patients with negative adenovirus tests reported having seasonal allergies, and $18 \%$ had bilateral cases. Additionally, during the COVID-19 era, several publications suggested an increased incidence of dry eye due to lockdown related environmental changes, including mask use, increased use of screens, and dietary deficiencies. $^{29-31}$ 
In our study cohort, $89 \%$ of patients presented with acute follicular conjunctivitis, usually suggesting a viral etiology. A majority of acute conjunctivitis patients usually present to primary care and urgent care. ${ }^{32}$ Since we conducted this study at a tertiary center, we postulated that the timing of the swab may have played a role in the lower rates of confirmed adenoviral conjunctivitis within our sample. By the time conjunctival swabs were collected, patients may have already been in the later stages of viral conjunctivitis, and swabs may have yielded a negative result because of this. Even though we avoided using preservative containing topical anesthetic drops before collecting samples, some patients reported using topical eye drops in the preceding days of the study visit. It is not clear whether these eye drops had an antiviral activity which may have been the reason for the zero-occurrence rate of SARS-CoV -2 associated conjunctivitis. ${ }^{33}$ Additionally, the sensitivity and specificity of RT-PCR for diagnosing SARS-CoV infection are $85-87 \%$ and $100 \%$, respectively. ${ }^{17}$ Thus, there may have been false-negative cases within our sample.

Is it important to know whether SARS-CoV-2 is a common reason for conjunctivitis during the pandemic? Treatment for conjunctivitis would not differ based on the viral etiology since there is no FDA-approved treatment for any viral conjunctivitis. However, it is crucial to identify the viral etiology to prevent its spread as both SARS$\mathrm{CoV}-2$ and adenovirus are very contagious and can cause respiratory disease. In a recent editorial on ocular surface and COVID-19, eye protection was explicitly recommended for health care workers and patients at risk, even though there is a low risk of SARS-CoV-2 spreading through tears. ${ }^{34}$ Currently, there is a lack of universally agreed recommendations to protect health care workers, specifically eye care providers, and allied ophthalmic personnel. Although ruling out common viral etiologies and testing patients for SARS-CoV-2 in ocular samples were recommended for early detection of subclinical COVID-19 cases to limit further spread, ${ }^{17}$ our data do not suggest that precautions to prevent viral spread when viral conjunctivitis is suspected should be different from what it was before the COVID-19 pandemic. We also suggest that it is probably not valuable to perform routine testing for SARS-CoV-2 in every patient presenting only with conjunctivitis.

The main limitation of our study was the small sample size. Larger sample size may have yielded a more accurate estimation of the prevalence of SARS-CoV-2 associated conjunctivitis. There may have been a selection bias due to conducting this study at a tertiary care institution and the willingness of patients to seek treatment. Some of the cases we enrolled in were referred by primary care providers or urgent care centers after failed initial treatment. This may have resulted in a lower positivity rate of confirmed cases, as discussed above. In addition, the results would have been influenced by location, strain, and local population prevalence. Our results should be interpreted considering the first year of the pandemic when different strain(s) was dominant compared to the subsequent year. Another limitation was that we collected samples only at one-time point. It is recommended to collect two samples two to three days apart to rule out viral etiology. ${ }^{17}$ We may have had falsenegative test results due to the timeline of sample collection. However, due to ethical reasons, we limited the number of in-person study visits to reduce the risk of exposure of study participants and protect others during the ongoing pandemic. Lastly, a few participants did not agree to provide nasal or nasopharyngeal swabs. Asymptomatic patients may test positive for SARS-CoV-2 in nasal or nasopharyngeal samples while testing negative in ocular samples. Although only three patients refused to provide neither nasal nor nasopharyngeal swabs, we may have missed relevant information.

\section{Conclusion}

Our study demonstrated that SARS-CoV-2 associated conjunctivitis was uncommon in the absence of COVID-19 even during the peak of the pandemic. We suggest that routine conjunctival testing is not valuable in patients with acute conjunctivitis in the absence of respiratory symptoms suggesting COVID-19. A multicenter study with a larger sample size would yield a more accurate estimation of the prevalence of SARS-CoV-2 conjunctivitis.

\section{Acknowledgments}

The statistical analysis was performed with the assistance of Wilmer Biostatistics Center supported by the NEI P30 Core Grant P30EY001765.

\section{Disclosure}

The authors report no conflicts of interest in this work.

\section{References}

1. World Health Organization. Report of the WHO-China joint mission on coronavirus disease 2019 (COVID-19); 2020. Available from: https://www.who.int/publications/i/item/report-of-the-who-china-jointmission-on-coronavirus-disease-2019-(covid-19). Accessed August 11, 2021. 
2. Wang D, Hu B, Hu C, et al. Clinical characteristics of 138 hospitalized patients with 2019 novel coronavirus-infected pneumonia in Wuhan, China. JAMA. 2020;323:1061-1069. doi:10.1001/ jama.2020.1585

3. Loffredo L, Pacella F, Pacella E, et al. Conjunctivitis and COVID-19: a meta-analysis. J Med Virol. 2020;92:1413-1414. doi:10.1002/ jmv. 25938

4. Guan W, Ni Z, Hu Y, et al. Clinical characteristics of coronavirus disease 2019 in China. N Engl J Med. 2020;382:1708-1720. doi:10.1056/NEJMoa2002032

5. Colavita F, Lapa D, Carletti F, et al. SARS-CoV-2 isolation from ocular secretions of a patient with COVID-19 in Italy with prolonged viral RNA detection. Ann Intern Med. 2020;173:242-243. doi:10.7326/M20-1176

6. Chen L, Liu M, Zhang Z, et al. Ocular manifestations of a hospitalised patient with confirmed 2019 novel coronavirus disease. Br J Ophthalmol. 2020;104:748-751. doi:10.1136/bjophthalmol-2020-316304

7. Li JO, Lam DSC, Chen Y, Ting DSW. Novel coronavirus disease 2019 (COVID-19): the importance of recognising possible early ocular manifestation and using protective eyewear. $\mathrm{Br}$ J Ophthalmol. 2020;104:297-298. doi:10.1136/bjophthalmol-2020315994

8. Wu P, Duan F, Luo C, et al. Characteristics of ocular findings of patients with coronavirus disease 2019 (COVID-19) in Hubei Province, China. JAMA Ophthalmol. 2020;138:575-578. doi:10.1001/jamaophthalmol.2020.1291

9. Xia J, Tong J, Liu M, et al. Evaluation of coronavirus in tears and conjunctival secretions of patients with SARS-CoV-2 infection. J Med Virol. 2020;92:589-594. doi:10.1002/jmv.25725

10. Scalinci SZ, Trovato Battagliola E. Conjunctivitis can be the only presenting sign and symptom of COVID-19. IDCases. 2020;20: e00774. doi:10.1016/j.idcr.2020.e00774

11. Ying NY, Idris NS, Muhamad R, Ahmad I. Coronavirus disease 2019 presenting as conjunctivitis. Korean J Fam Med. 2021;42:487-490. doi: $10.4082 / \mathrm{kjfm} .20 .0090$

12. Corman VM, Landt O, Kaiser M, et al. Detection of 2019 novel coronavirus (2019-nCoV) by real-time RT-PCR. Euro Surveill. 2020;25:2000045. doi:10.2807/1560-7917.ES.2020.25.3.2000045

13. Zhang X, Chen X, Chen L, et al. The evidence of SARS-CoV-2 infection on ocular surface. Ocul Surf. 2020;18:360-362. doi:10.1016/j.jtos.2020.03.010

14. Cavalleri M, Brambati M, Starace V, et al. Ocular features and associated systemic findings in SARS-CoV-2 infection. Ocul Immunol Inflamm. 2020;28:916-921. doi:10.1080/09273948.20 20.1781198

15. Ya Y, Yanping S, Ming Y, et al. Novel coronavirus pneumonia combined with conjunctivitis: three cases report. Chin J Exp Ophthalmol. 2020;38:242-244.

16. Qu J, Xie H, Zhang M. Evidence of SARS-CoV-2 transmission through the ocular route. Clin Ophthalmol. 2021;15:687-696. doi: $10.2147 /$ OPTH.S295283

17. Ankita AK, Saxena SK. COVID-19: an ophthalmological update. In: Saxena SK, editor. Coronavirus Disease 2019 (COVID-19): Epidemiology, Pathogenesis, Diagnosis, and Therapeutics. Singapore: Springer Singapore; 2020:81-93.
18. Deng W, Bao L, Gao H, et al. Ocular conjunctival inoculation of SARS-CoV-2 can cause mild COVID-19 in rhesus macaques. Nat Commun. 2020;11:4400. doi:10.1038/s41467-020-18149-6

19. Dutescu RM, Banasik P, Schildgen O, et al. Detection of coronavirus in tear samples of hospitalized patients with confirmed SARS-CoV-2 from oropharyngeal swabs. Cornea. 2021;40:348-350. doi:10.1097/ ICO.0000000000002562

20. Huang C, Wang Y, Li X, et al. Clinical features of patients infected with 2019 novel coronavirus in Wuhan, China. Lancet. 2020;395:497-506. doi:10.1016/S0140-6736(20)30183-5

21. Zhou Y, Duan C, Zeng Y, et al. Ocular findings and proportion with conjunctival SARS-COV-2 in COVID-19 patients. Ophthalmology. 2020;127:982-983. doi:10.1016/j.ophtha.2020.04.028

22. Garcia-Zalisnak D, Rapuano C, Sheppard JD, Davis AR. Adenovirus ocular infections: prevalence, pathology, pitfalls, and practical pointers. Eye Contact Lens. 2018;44(Suppl 1):S1-S7. doi:10.1097/ ICL.0000000000000226

23. Azari AA, Barney NP. Conjunctivitis: a systematic review of diagnosis and treatment. JAMA. 2013;310:1721-1729. doi:10.1001/ jama.2013.280318

24. Singh RB, Liu L, Anchouche S, et al. Ocular redness - I: etiology, pathogenesis, and assessment of conjunctival hyperemia. Ocul Surf. 2021;21:134-144. doi:10.1016/j.jtos.2021.05.003

25. Pekel G, Azman E, Pekel E, et al. Preocular tear film tests in acute hemorrhagic conjunctivitis caused by coxsackievirus A24 variant. Turk J Ophthalmol. 2012;42:186-189.

26. Wright PW, Strauss GH, Langford MP. Acute hemorrhagic conjunctivitis. Am Fam Physician. 1992;45:173-178.

27. $\mathrm{Li} \mathrm{W}$, Wong $\mathrm{S}, \mathrm{Li} \mathrm{F}$, et al. Animal origins of the severe acute respiratory syndrome coronavirus: insight from ACE2-S-protein interactions. $J$ Virol. 2006;80:4211-4219. doi:10.1128/ JVI.80.9.4211-4219.2006

28. Bielory BP, O'Brien TP, Bielory L. Management of seasonal allergic conjunctivitis: guide to therapy. Acta Ophthalmol. 2012;90:399-407. doi:10.1111/j.1755-3768.2011.02272.x

29. Napoli PE, Nioi M, Fossarello M. The "Quarantine Dry Eye": the lockdown for coronavirus disease 2019 and its implications for ocular surface health. Risk Manag Healthc Policy. 2021;14:1629-1636. doi:10.2147/RMHP.S277067

30. Saldanha IJ, Petris R, Makara M, Channa P, Akpek EK. Impact of the COVID-19 pandemic on eye strain and dry eye symptoms. Ocul Surf. 2021;22:38-46. doi:10.1016/j.jtos.2021.06.004

31. Prescott CR. Increased screen time and dry eye: another complication of COVID-19. Eye Contact Lens. 2021;47:433. doi:10.1097/ ICL.0000000000000820

32. Kaufman HE. Adenovirus advances: new diagnostic and therapeutic options. Curr Opin Ophthalmol. 2011;22:290-293. doi:10.1097/ ICU.0b013e3283477cb5

33. Napoli PE, Mangoni L, Gentile P, Braghiroli M, Fossarello M. A panel of broad-spectrum antivirals in topical ophthalmic medications from the drug repurposing approach during and after the coronavirus disease 2019 era. J Clin Med. 2020;9:2441. doi:10.3390/ jem9082441

34. Napoli PE, Nioi M, d'Aloja E, Fossarello M. The ocular surface and the coronavirus disease 2019: does a dual "ocular route" exist? J Clin Med. 2020;9:1269. doi:10.3390/jcm9051269 


\section{Publish your work in this journal}

Clinical Ophthalmology is an international, peer-reviewed journal covering all subspecialties within ophthalmology. Key topics include: Optometry; Visual science; Pharmacology and drug therapy in eye diseases; Basic Sciences; Primary and Secondary eye care; Patient Safety and Quality of Care Improvements. This journal is indexed on PubMed

Submit your manuscript here: https://www.dovepress.com/clinical-ophthalmology-journal
Central and CAS, and is the official journal of The Society of Clinical Ophthalmology (SCO). The manuscript management system is completely online and includes a very quick and fair peer-review system, which is all easy to use. Visit http://www.dovepress.com/ testimonials.php to read real quotes from published authors. 